\title{
ANÁLISE ESPACIAL DA DESIGUALDADE ECONÔMICA NA REGIÃO NORTE DO BRASIL: UM ESTUDO DAS MICRORREGIÕES
}

\author{
Spatial Analysis of Economic Inequality in Northern Brazil: A Study of Microregions
}

Henry Antônio Silva Nogueira ${ }^{1}$ Rubicleis Gomes da Silva²

\begin{abstract}
RESUMO: O objetivo geral deste trabalho é identificar padrões espaciais de desigualdade econômica intermunicipal entre as microrregiões que compõem a região Norte do Brasil no período de 2010. Especificamente, pretende-se: a) determinar o grau de desigualdade econômica intermunicipal para as microrregiões, b) verificar se existe dependência espacial entre as microrregiões e, por fim, c) identificar o relacionamento entre as variáveis PIB per capita e desigualdade, e entre o Índice de Desenvolvimento Humano Municipal (IDH-M) e desigualdade econômica. Para alcançar os objetivos propostos, é utilizado o cálculo do grau de desigualdade de Williamson (coeficiente de Williamson) para as microrregiões, e ainda, são utilizadas técnicas de análise de distribuição espacial, especificamente, a Análise Exploratória de Dados Espaciais (AEDE), juntamente com as medidas de auto correlação I de Moran global e local, e seus respectivos índices. O estudo confirma observações empíricas e revela que na Região norte do Brasil ocorre um alto grau de desigualdade entre as microrregiões que a compõem. A desigualdade econômica é menor quando se aplica a análise às microrregiões. Ficou claro que existe um padrão de distribuição do grau de desigualdade entre as microrregiões do Norte do país, ou seja, em certa medida, pode-se afirmar que em alguns casos ocorre dependência pendencia espacial.
\end{abstract}

Palavras-chave:Desigualdade econômica, microrregião,AEDA.

ABSTRACT: The aim of this work is to identify spatial patterns of economic inequality between the inter micro comprising the northern region of Brazil from 2010. Specifically, we intend to: a) determine the degree of economic inequality intermunicipal for micro, b) check whether there is spatial dependence between the micro and finally, c) identify the relationship between the variables GDP per capita and inequality, between the Municipal Human Development Index (HDI-M) and economic inequality. To achieve the proposed objectives, is used to calculate the degree of inequality Williamson (Williamson coefficient) for micro- and still are used technical analysis of spatial distribution, specifically, Exploratory Spatial Data Analysis (ESDA), along with measures of autocorrelation Moran's I global and local, and their respective indices. The study confirms empirical observations and reveals that in the Northern region of Brazil is a high degree of inequality between the microregions that make up. Economic inequality is smaller when applying the analysis to the micro. It was clear that there is a pattern of distribution of the degree of inequality between micro North of the country, ie, to a certain extent, it can be said that in some cases pending spatial dependence occurs.

Keywords: Economic inequality, Microregions, AEDA.

JEL: R00

\footnotetext{
${ }^{1}$ Economista. Controlador Interno do Tribunal de Contas do Estado do Acre. Mestre em Desenvolvimento Regional pela Universidade Federal do Acre.pr_henry@hotmail.com

${ }^{2}$ Economista. Doutor em Economia Ap licada e Professor Adjunto de Métodos Quantitativos da Universidade Federal do Acre.rubicleis@uol.com.br
} 


\section{INTRODUÇÃO}

A desigualdade econômica no espaço geográfico é abordada de forma mais intensa a partir da década de 1950, com as chamadas teorias da localização e do desenvolvimento desigual. Mas, recentemente, a análise do espaço geográfico e sua influência no desempenho econômico das regiões têm ocupado lugar de destaque na teoria econômica por meio da chamada Nova Geografia Econômicas (NGE), que formaliza o papel dos retornos crescentes de escala para o crescimento econômico e a distribuição da atividade econômica no espaço.

Dentre os vários determinantes da geração de riqueza, a desigualdade na distribuição das atividades produtivas, bem como sua influência no espaço geoeconômico tem chamado atenção. Neste sentido, é de suma importância conhecer os fatores que afetam o nível de desigualdade de uma determinada economia regional. A compreensão de como fatos econômicos de uma região se relaciona com os observados nas demais regiões vizinhas, constitui um dado cada vez mais relevante na tentativa de se explicar as particularidades regionais.

No caso do Brasil, a desigualdade na distribuição da atividade econômica no espaço constitui uma das características mais prevalecentes. Mesmo com bom desempenho da economia nos últimos anos, as mudanças estruturais não foram capazes de alterar significativamente o quadro da desigualdade econômica inter-regional. Atualmente, o Brasil é uma República Federativa composta pela União, por 26 estados mais o Distrito Federal e por 5.565 municípios. Seu sistema federativo foi adotado a partir de 1889, com a Proclamação da República, que transformou as províncias em estados (ANDRADE; ANDRADE, 2003).

Além dessa divisão político-administrativa, objetivando um melhor conhecimento do território nacional, existem ainda, divisões regionais, na qual de forma mais agregada se encontram as denominadas grandes regiões Norte, Nordeste, Sul Sudeste e Centro-Oeste, que se constituem em agrupamentos de estados. Comumente, os estudos a respeito da desigualdade econômica regional utilizam dados de regiões agregadas, geralmente, por estados ou grandes regiões. Porém, a desigualdade espacial da atividade econômica pode ser observada não somente entre as regiões com maiores níveis de agregação, mas também entre microrregiões inscritas no território.

A dinâmica do processo de desenvolvimento capitalista no Brasil se reflete na alta desigualdade econômica observada na organização espacial do país, o qual comporta diferenciadas formas de subordinação da força de trabalho ao capital e pela presença crescente das ações de intervenção do Estado na economia.

Neste sentido, pressupõe-se que as desigualdades econômicas devem ser minimizadas não somente ao nível das grandes regiões geográficas, mas também nas microrregiões, haja vista que o processo de desenvolvimento e crescimento econômico deve ocorrer da forma mais homogênea no espaço, se expandido e refletido um melhor bem-estar das comunidades locais.

Revista de Estudos Sociais | Ano 2015, N. 33, V. 17, Pag. 29 
Sendo assim, este estudo tem sua abrangência geográfica limitada à região Norte do Brasil. A escolha dessa região deve-se a sua grande extensão territorial que corresponde a $42,27 \%$ do território brasileiro, além do fato de que, apesar do bom desempenho econômico dos últimos anos, essa é uma região caracterizada por fortes desigualdades sociais e econômicas evidenciadas nas elevadas taxas de pobreza e altos índices de concentração da riqueza.

Mais especificamente se estuda as subdivisões regionais denominadas microrregiões que compõem a região Norte do país. Conforme o Instituto Brasileiro de Geografia e Estatística - IBGE(2011), essas microrregiões foram definidas, em 1990, como "um conjunto de municípios, contíguos e contidos na mesma unidade da federação (estados), definidos com base em características do quadro natural, da organização da produção e de sua integração".

Pretende-se responder a seguinte indagação: existe um padrão espacial da desigualdade econômica intermunicipal entre as microrregiões do Norte do Brasil?

A resposta a essa pergunta proporcionará o conhecimento do cenário da desigualdade em nível de agregação regional pouco estudado, as microrregiões. Além disso, o problema levantado tem muita importância, haja vista que a definição geográfica de um padrão espacial da desigualdade entre as microrregiões estudadas contribuirá para estudos mais aprofundados a respeito da desigualdade na região Norte.

O uso de modelos de econometria espacial tem-se baseado, em sua maioria, em dados dos estados brasileiros. Magalhães et al. (2000) que utilizam técnicas de econometria espacial para investigar o processo de convergência de renda entre os estados brasileiros, no período 1970-1995. Os resultados mostram a existência de dependência espacial entre os estados brasileiros e sugerem, portanto, que modelos de crescimento econômico que utilizam dados dos estados brasileiros e ignoram a dependência espacial estão mal especificados. Pimentel e Haddad (2004) analisam a renda do trabalho por habitante, para o ano de 2000, nos setores agropecuário, industrial e de serviços. Utilizando dados microrregionais do estado de Minas Gerais, os autores identificam um padrão espacial para os setores analisados e encontram clusters com padrões alto-alto (regiões com elevados níveis de renda por habitantes cercadas por regiões de altos níveis de renda por habitante) na parte oeste do estado; e baixo-baixo (regiões com baixos níveis de renda por habitante cercadas de regiões com igual desempenho) na parte nordeste do estado.

O objetivo geral, desse trabalho é identificar padrões espaciais de desigualdade econômica intermunicipal entre as microrregiões que compõem a região Norte do Brasil no período de 2010. Especificamente, pretende-se: a) determinar o grau de desigualdade econômica intermunicipal para as microrregiões, b) verificar se existe dependência espacial entre as microrregiões e, por fim, c) identificar o relacionamento entre as variáveis PIB per capita e desigualdade econômica, e entre o Índice de Desenvolvimento Humano Municipal (IDH-M) e desigualdade econômica.

Segue-se a hipótese de que a desigualdade econômica intermunicipal entre as microrregiões do Norte brasileiro não apresenta um padrão espacial de distribuição. Para alcançar os objetivos propostos, é utilizado o cálculo do grau 
de desigualdade de Williamson (coeficiente de Williamson) para as microrregiões, e ainda, são utilizadas técnicas de análise de distribuição espacial, especificamente, a Análise Exploratória de Dados Espaciais (AEDE), juntamente com as medidas de auto correlação I de Moran global e local, e seus respectivos índices.

Além desta seção, a próxima destaca aspectos econômicos e populacionais. Em seguida tem-se a metodologia aplicada que descreve os referenciais teóricos e analíticos. Nas seções posteriores se encontra a fonte dos dados utilizados, resultados e discussões, seguidos pelas considerações finais do estudo.

\section{População e PIB do Norte do Brasil}

De acordo do IBGE (2012) no período compreendido entre 2000 e 2010, primeira década do século XXI, a região Norte brasileira apresentou taxa média de crescimento anual da população de 2,09\%, ficando acima da média nacional. A população total brasileira cresceu em média no mesmo período 1,17\%.

Em relação aos aspectos econômicos, de forma geral, a região apresentou o bom desempenho econômico na primeira década de 2000. Considerando o PIB em valores correntes, a Tabela 1 mostra que a região obteve crescimento médio anual a taxa de $14,57 \%$, acima do verificado em nível nacional de $12,32 \%$.

Tabela 1 - Taxa média de crescimento anual do PIB, segundo as grandes regiões nos anos de 2000 a 2010

Brasil/Grandes regiões

Taxa média de crescimento anual

do $\mathrm{PIB}^{\star}(\%)$

\begin{tabular}{ll}
\hline Brasil & 12,32 \\
Norte & $\mathbf{1 4 , 5 7}$ \\
Nordeste & 13,20 \\
Sudeste & 11,74 \\
Sul & 12,34 \\
Centro-Oeste & 13,48
\end{tabular}

Fonte: IBGE (2012). Censos demográficos 2000/2010.

*Valores correntes.

$\mathrm{Na}$ comparação com o crescimento médio anual do PIB das demais grandes regiões, o Norte obteve o melhor resultado, ou seja, taxa crescimento médio do PIB acima de todas as obtidas nas outras grandes regiões do país. As migrações de mão-de-obra especializada para região e o investimento público são fatores que podem explicar o impacto positivo na produtividade regional.

Revista de Estudos Sociais | Ano 2015, N. 33, V. 17, Pag. 31 
A região Norte brasileira constitui uma economia caracterizada por muitas fragilidades, entre elas, o baixo nível de industrialização e inovação tecnológica, infraestrutura deficitária e a grandes dificuldades de investimentos. Neste sentido, apesar do otimismo, de acordo com os dados da Tabela 2, o Produto Interno Bruto regional, no ano de 2010, representou apenas 5,3\% do PIB nacional. A região ocupa o último lugar no ranking da participação das grandes regiões no Produto Interno Bruto do país.

Tabela 2 - Participação das grandes regiões brasileiras no PIB nacional em 2010

\begin{tabular}{|c|c|c|}
\hline Brasil/Grandes regiões & 2010 & Ranking \\
\hline Brasil & 100 & - \\
\hline Norte & 5,3 & 50 \\
\hline Nordeste & 13,5 & $3^{\circ}$ \\
\hline Sudeste & 55,4 & 10 \\
\hline Sul & 16,5 & $2^{\circ}$ \\
\hline Centro-Oeste & 9,3 & 40 \\
\hline
\end{tabular}

Fonte: IBGE (2011).

Atualmente, a região Norte do Brasil é subdividida em um total de 64 microrregiões geográficas, agrupadas nas sete unidades da federação (estados) que compõem o território regional. A organização do espaço em microrregiões considerou interação entre as áreas que se relacionam em nível local. Neste sentido, a estrutura da produção utilizada como parâmetro para a determinação das microrregiões, tem forte relação com a produção propriamente dita, distribuição, trocas e consumo, incluindo as áreas urbanas e rurais.

A microrregião Belém, situada no estado do Pará, composta por seis municípios (dentre eles a capital do estado), apresentou a maior participação na população regional, perfazendo um total de $13,50 \%$ dos habitantes da região Norte. A microrregião Manaus, no estado do Amazonas, com sete municípios (dentre eles a capital do estado), representa $12,86 \%$ do total dos residentes do Norte do Brasil. As microrregiões Amapá e Oiapoque, compostas por três e dois municípios, respectivamente, ambas no estado do Amapá, constituem as que apresentaram menor participação na população regional, $0,16 \%$ e $0,18 \%$, respectivamente.

Ainda de acordo com dados do IBGE (2011), no ano de 2010, a microrregião Manaus contribui com a maior parcela do PIB da grande região Norte, representando $24,91 \%$ do produto regional. Em seguida, tem-se a microrregião Belém que representa $13,15 \%$ do PIB total. As microrregiões que apresentaram menor participação foram, Japurá, no estado do Amazonas, composta por dois municípios, que representou $0,06 \%$ do PIB regional e; a microrregião Amapá que representou $0,12 \%$ do PIB total. 


\section{ASPECTOS TEÓRICOS E METODOLÓGICOS}

\subsection{Referencial Teórico}

Para analisar de que maneira uma determinada região impacta nos resultados de outra é necessário utilizar-se de arcabouço teórico que versa sobre as externalidades. Desse modo, é importante salientar que a teoria das externalidades econômicas tem origem no âmbito da microeconomia, em geral, compreendendo a relação entre variáveis ou agentes econômicos.

Pindyck e Rubinfeld (1994 apud SILVA et al., 2009) destacam que na medida em que os efeitos de variáveis exógenas influenciam variáveis endógenas, sem que haja a devida compensação por parte dos agentes, têm-se a ocorrência de externalidades. Eaton e Eaton (1999) dispõem que em situações nas quais o comportamento de determinado agente econômico exerce impacto sobre outro agente econômico sem que ocorram as devidas compensações, pode-se dizer que existem externalidade na relação.

Longo (1993) conceitua a externalidade como uma imposição de um efeito externo causado por terceiros, gerada por meio de uma relação entre variáveis. Assim sendo, estes efeitos poderão ser positivos ou negativos, ou seja, externalidade positiva ou negativa.

Baldwin e Martin (2004) destacam a importância da análise do impacto das externalidades quanto à redução das desigualdades regionais. Segundo os autores, a concentração espacial da atividade econômica pode ter impactos dinâmicos positivos para as regiões menos desenvolvidas, ou seja, promover externalidades positivas. Isto ocorre, pelo fato de que quando as atividades econômicas se aglomeram, por exemplo, em uma região faz com que a economia como um todo se beneficie das externalidades locais.

Esse fenômeno é explicado haja vista que o produto agregado da economia regional cresceria a taxas mais elevadas, levando-se em consideração o fato de que o centro econômico cresceria a taxas elevadas, neste caso, a periferia se beneficiaria deste crescimento, tanto em termos de exportações como na transferência de renda, o que influência positivamente o cenário da desigualdade.

A evolução da desigualdade em uma determinada região pode, por exemplo, ser influenciada pela evolução da desigualdade e de outras variáveis nas regiões vizinhas, através de externalidades espaciais.

Varian (1992 apud SILVA et al., 2009) destaca que as atividades geradoras de externalidades não se encontram no estado do ótimo de Pareto $^{3}$, haja vista que na realização de tais atividades por determinado agente existem efeitos positivos ou negativos nas atividades desenvolvidas por outros agentes.

${ }^{3}$ Conceito de economia desenvolvido pelo italiano Vilfredo Pareto. Constitui uma situação ótima no sentido de Pareto se não for possível melhorar a situação, ou, mais genericamente, a utilidade de um agente, sem degradar a situação ou utilidade de qualquer outro agente.

Revista de Estudos Sociais | Ano 2015, N. 33, V. 17, Pag. 33 
De forma geral, existem externalidades quando as influências das ações externas não são compensadas, ou seja, ela ocorre quando os benefícios ou malefícios causados pelo comportamento não são incorporados no processo. Neste contexto, determinada região pode sofrer externalidades disseminadas de outras regiões, acarretando inclusive em relação de dependência espacial.

Analisando a Figura 2.1, é possível visualizar o ponto ótimo de externalidade da desigualdade de uma região sobre outra. $O$ eixo vertical representa os efeitos negativos (custos) ou positivos (benefícios); o eixo horizontal mostra o grau de desigualdade econômica, em que $Q 1$ representa o nível ótimo de externalidade e Q2, o nível de externalidade associado com a maximização do lucro em concorrência perfeita.

\subsection{Referencial Analítico}

\subsubsection{O Grau de Desigualdade Regional}

Inicialmente, para determinar o grau de desigualdade econômica nas mesorregiões do Norte do Brasil, utiliza-se neste trabalho o cálculo do Coeficiente de Williamson $\left(V_{w}\right)$ desenvolvido por Williamson (1965) que expressa um grau de desigualdade econômica entre diferentes regiões. De acordo com Williamson (1965) os valores relativos ao Coeficiente de Williamson, que mensuram o grau de desigualdade econômica regional, são obtidos pela equação $0^{4}$

$$
V w=\frac{\sqrt{\sum_{i=1}^{n}\left(P I B_{p c, i}-P I B_{p c, E}\right)^{2} \frac{P_{i}}{P_{E}}}}{P I B_{p c, E}}
$$

em que $V_{w}$ corresponde ao coeficiente de desigualdades regionais (grau de desigualdade); $P I B_{p c, i}$, éproduto interno bruto per capita da região menor; $P I B_{p c, E}$, produto interno brutoper capita da região maior; $P_{i}$, população i-ésima da região menor; e $P_{E}$, população da região maior.

O valor de $V_{w}$ varia entre o máximo de 1 (um) e o mínimo de 0 (zero), sendo que quanto mais próximo da unidade, maiores serão as desigualdades econômicas regionais. O Coeficiente de Desigualdade regional formulado por Williamson tem como principais parâmetros o produto interno bruto e a população regional.

\subsubsection{A Análise Exploratória de Dados Espaciais}

Fotheringham et al. (2002 apudALMEIDA, 2012) recomendam que antes da determinação de qualquer modelo espacial se faz necessário, a priori, uma análise exploratória de dados espaciais (AEDE).

${ }^{4} \mathrm{O}$ modelo original de Williamson (1965) usava a renda do trabalhador. Esta variável foi substituída neste trabalho pelo PIB das regiões. A adaptação foi realizada a fim de adequar a formula de cálculo do $V w$ a este trabalho. 
Em Ramos (2002), a dependência espacial pode ser entendida como a tendência a que o valor de uma variável associada a uma determinada localização assemelhe-se mais ao valor de suas amostras vizinhas do que ao restante das localizações do conjunto amostral. O conceito de vizinhança adotado pode ser formulado tanto com base na proximidade, (zonas fronteiriças, distância linear etc.), como também na similaridade de outras características regionais.

A dependência espacial, segundo Almeida (2004), é dada pela interação dos agentes nos espaços, ou seja, o valor de uma variável de interesse numa certa região $i$ depende do valor dessa variável nas regiões vizinhas $j$. A inserção da localização no estudo é importante, pois quando este não é inserido os resultados proporcionados pela econometria convencional podem se tornar inválidos. A inserção de técnicas espaciais incorpora na modelagem o padrão da interação socioeconômica entre os agentes do sistema, bem como as características da estrutura desse sistema no espaço.

A heterogeneidade espacial manifesta-se quando ocorre a instabilidade estrutural no espaço. Dessa forma, há diferentes respostas dependendo da localidade ou da escala espacial. A heterogeneidade espacial ocorre na situação em que os coeficientes ou os padrões do erro variam sistematicamente através das áreas geográficas.

Conforme destacado por Almeida (2004), nos processos espaciais existe um imbricamento entre os dois efeitos, pois a heterogeneidade espacial gera dependência espacial e, por sua vez, a dependência espacial pode levar a heterogeneidade.

Segundo Anselin (1999 apud ALMEIDA, 2012), a Analise Exploratória de Dados Espaciais (AEDE) é uma coleção de técnicas para descrever e visualizar distribuições espaciais, identificar localidades atípicas, descobrir padrões de associação espacial e sugerir diferentes regimes espaciais ${ }^{5}$ e outras formas de instabilidade espacial.

O objetivo desse método é apresentar distribuições espaciais, padrões de associação (clusters espaciais), identificar existência de regimes espaciais ou outras formas de instabilidade espacial, bem como identificar observações atípicas (outliers). Os indicadores da análise exploratória são ferramentas utilizadas não somente para caracterização do espaço, mas ainda, para avaliar a distribuição relativa do arranjo espacial dos eventos (CARVALHO, 2009).

Carvalho (2009) destaca ainda, que os indicadores que abrangem todas as observações da autocorrelação espacial são conhecidos como estatísticas globais, e os que avaliam a autocorrelação espacial para cada observação específica são denominados de estatísticas locais.

$\mathrm{Na}$ análise de dados espaciais, primeiramente, deve-se testar a hipótese de que os dados são distribuídos de forma aleatória. Para verificar se uma variável em determinada região se relaciona com resultados da mesma variável de outras regiões Anselin (2003), propõe o teste de autocorrelação de I de Moran

${ }^{5}$ Conforme Anselin (2005), regimes espaciais são sub-regiões que mostram distintas distribuições para dada variável.

Revista de Estudos Sociais | Ano 2015, N. 33, V. 17, Pag. 35 
univariado global. De acordo com Martino (2005) o I de Moran univariado é definido por:

$$
I_{t}=\left(\frac{n}{S_{0}}\right)\left(\frac{Z_{t}^{\prime} W Z_{t}}{Z_{t}^{\prime} Z_{t}}\right)
$$

em que $I_{t}$ é o teste de auto correlaçãouni variado global; $t=1, \ldots, \mathrm{n} ; \mathrm{Z}, \mathrm{o}$ vetor de $\mathrm{n}$ observações para 0 ano $t$, na forma de desvio padrão em relação à média; $W$, a matriz de pesos espaciais; $S_{0}$, a soma de todos os elementos de $W$.

Para verificar se uma variável em determinada região se relaciona com resultados das outras variáveis no espaço utiliza-se do teste de auto correlação I de Moran multivariado, que é dado por:

$$
I=\frac{n}{S} \frac{\sum_{i} \sum_{j} w_{i j}\left(x_{i}-u\right)\left(x_{j}-u\right)}{\sum_{i}\left(x_{i}-u\right)^{2}}
$$

em que lé o teste de auto correlação multivariado global; n, o número de observações; $x_{i}$ e $x_{j}$, as observações nas localidades i e j (com média $u$ ); $w$, a matriz de pesos; e $\mathrm{S}$, o escalar constante dado pela $S=\sum_{i} \sum_{j} w_{i j}$.

Em essência, o indicador equivale ao grau de associação linear entre o vetor de valores observados (z) e a média ponderada de valores vizinhos $(\mathrm{Wz})$. Valores maiores (menores) que os esperados indicam autocorrelação espacial positiva (negativa).

Quando a normalização dos pesos nas linhas da matriz das distâncias é efetuada, o que é preferível (ANSELIN, 1995), $S$ iguala $n$, uma vez que os pesos de cada linha somados devem ser iguais à unidade, e o teste estatístico é comparado com a sua média teórica, $l=-1 /(n-1)$. Então $I$ tende a 0 , quando $n$ tende a 0 . A hipótese nula $\mathrm{HO}: \mathrm{I}=-1 /(\mathrm{n}-1)$ é testada contra a hipótese alternativa $H 1: \mid \neq-1 /(n-1)$.

Quando H0 é rejeitada e $1>-1 /(n-1)$ verifica-se a existência de autocorrelação espacial positiva. Isto é, os valores altos e os valores baixos estão mais espacialmente "clustered" do que se poderia assumir puramente por acaso. Se H0 é novamente rejeitada, mas $\mid<-1 /(n-1)$ isto indica autocorrelação espacial negativa.

De forma geral, o índice de Moran presta-se a um teste cuja hipótese nula é de independência espacial; neste caso, seu valor seria zero. Valores positivos (entre 0 e +1) indicam para correlação direta e negativos, (entre 0 e -1 ) correlação inversa. Uma vez calculado, é importante estabelecer sua validade estatística. Em outras palavras, será que os valores medidos representam correlação espacial significativa? 
Para estimar a significância do índice, será preciso associar a este uma distribuição estatística, sendo mais usual relacionar a estatística de teste à distribuição normal (MARTINO, 2005).

Uma das ferramentas mais usadas para verificar esta estrutura é o indicador local de associação espacial, conhecidos como LISA. Estes são usados para testar a hipótese nula de distribuição aleatória por comparar os valores de cada localização específica com os valores de seus vizinhos.

Por meio do LISA é possível classificar as associações espaciais locais entre as microrregiões e seus vizinhos de acordo com os seguintes resultados:

Padrão Alto-Alto (AA): revela microrregiões com alto valor da variável sob análise, cercada de vizinhos que também possuem valores semelhantes (quadrante superior direito);

Padrão Baixo-Baixo (BB): revela microrregiões com baixo valor circundadas por outras de valores também baixos (quadrante inferior esquerdo);

Padrão Alto-Baixo (AB): aponta microrregiões de alto valor que possuem vizinhos com baixo valor (quadrante inferior direito);

Padrão Baixo-Alto (BA): aponta microrregiões de baixo valor que são vizinhas de outras com alto valor (quadrante superior esquerdo).

Os padrões AA e BB revelam associação espacial positiva, enquanto que $B A$ e $A B$, associação espacial negativa.

Para examinar os padrões de dados espaciais em uma escala de maior detalhe, quando a dependência espacial é mais acentuada, é recomendado o Indicador Local de Associação Espacial (LISA), que produz um valor específico para cada objeto, permitindo assim, a identificação de agrupamentos de objetos com valores de atributos semelhantes ou objetos anômalos (LORENA, 2011).

Segundo Anselin (1995), indicadores LISA devem propiciar uma medida do grau em que o agrupamento espacial de valores locais similares é significativo. Neste caso se utiliza do I de Moran local, definido como segue:

$$
I_{i}=\frac{x_{i}-\bar{x}}{S_{i}^{2}} \sum_{j=1}^{N} w_{i j}\left(x_{i}-\bar{x}\right) \text { em que } S_{i}^{2}=\frac{\sum_{j=1, j \neq i}^{N} x_{j}^{2}}{N-1}-\bar{x}^{2}
$$

em que $l_{i}$ é o indicador de autocorrelação local; $x$, a variável observada; $x$, a média das observações; $N$, o total de observações.

Martino (2005) destaca que o teste de autocorrelação I de Moran local investiga se os valores vindos do teste de autocorrelação global são significantes ou não. Silva et al. (2006) afirmam que a interpretação do teste I de Moran local e da hipótese nula são as mesmas verificadas para I de Moran Global.

A partir disso, tem-se a seguinte classificação: alto-alto, para agrupamentos regiões que apresentam altos valores da variável; baixo-baixo,

Revista de Estudos Sociais | Ano 2015, N. 33, V. 17, Pag. 37 
para agrupamentos regionais que apresentam baixos valores da variável; baixoalto ou alto-baixo, para agrupamentos regionais que apresentam valores inversos.

Neste trabalho, utilizou-se da matriz de pesos espaciais do tipo rainhae torre. A conversão de contiguidade do tipo Rainha classifica como vizinhos todos os municípios que apresentam fronteira física comum ("norte-sul, leste-oeste"), e também vértices comuns. Já a matriz de peso espacial do tipo Torre considera como contíguos os municípios que apresentam, apenas, fronteiras (físicas) comuns.

\subsection{Fonte de Dados}

Os dados referentes ao Produto Interno do Bruto (PIB) e população dos municípios, bem como os relativos aos totais gerais das microrregiões, foram extraídos do Sistema IBGE de Recuperação Automática (SIDRA). O Sistema é disponibilizado pelo Instituto Brasileiro de Geografia e Estatística (IBGE), com o objetivo de facilitar aos administradores públicos e à sociedade em geral, através da Internet, a obtenção gratuita dos dados agregados de estudos e pesquisas realizados pelo por aquele órgão oficial.

No SIDRA é possível consultar dados na forma de séries temporais, analisando seu comportamento ao longo do tempo, bem como ter os mesmos disponibilizados por níveis territoriais desagregados, no presente caso foram utilizados dados de município, estados e grande região do Brasil.

O grau de desigualdade utilizado neste trabalho é determinado pelo Coeficiente de Willianson calculado para microrregiões do Norte do Brasil a partir dos municípios que as compõem.

\section{RESULTADOS E DISCUSSÃO}

\subsection{O Grau de Desigualdade Econômica entre as Microrregiões do Norte do Brasil}

Nesta seção, a partir de uma análise das microrregiões da região Norte brasileira, trata-se especificamente do grau de desigualdade econômica mostrado pelo Coeficiente de Williamson, para o período de 2010. Inicialmente, é analisada a desigualdade ao nível mais agregado de microrregiões, ou seja, a desigualdade entre todas as microrregiões da região Norte. Após a análise mais agregada, segue-se com a verificação do grau de desigualdade econômica entre as microrregiões por estado.

O grau de desigualdade econômica entre todas as microrregiões estudadas para o ano de 2010 foi de 0,76 (Anexo - Tabela 1A). Esse resultado indica que são grandes as distorções econômicas entre as microrregiões do Norte do Brasil. Esse fenômeno está relacionado diretamente com o fato de que a riqueza produzida na região Norte continua concentrada no espaço geográfico. 
A Tabela 3 mostra o grau de desigualdade econômica entre as microrregiões por estado da região Norte. Verifica-se que o Estado do Pará apresenta um alto grau de desigualdade econômica entre suas microrregiões, igual a 0,96 , ou seja, neste estado ocorre forte concentração de atividades econômicas em poucas microrregiões do estado. O segundo ponto a ser destacado, é o grau de desigualdade econômica de 0,52 , determinado para 0 estado do Amazonas. Esse foi o segundo mais alto resultado entre os demais estados analisados.

Ainda que menor intensidade, assim como no estado do Pará, a desigualdade econômica entre as microrregiões do Amazonas é relativamente alta, haja vista que se evidencia também uma forte concentração da atividade econômica em poucos municípios e, por conseguinte nas microrregiões.

Conforme mostrado na Tabela 3, os demais Estados apresentaram graus de desigualdade relativamente baixos. O Estado do Amapá foi o que apresentou o resultado mais positivo no quadro da desigualdade econômica. O grau de desigualdade entre as microrregiões do Amapá foi de 0,11 , o que indica uma forte similaridade na participação das microrregiões na formação do PIB do estado.

Seguindo o estado do Amapá, observa-se que os Estados de Rondônia, Acre, Roraima e Tocantins apresentam baixos índices de desigualdade econômica entre as microrregiões. Esses estados apresentam forte similaridade em relação à participação de suas microrregiões no PIB estadual.

Tabela 3 - Grau de desigualdade econômica entre as microrregiões por estado da região Norte do Brasil, em 2010

\begin{tabular}{lcc}
\hline \multicolumn{1}{c}{ UF } & $\begin{array}{c}\text { Quantidade } \\
\text { de } \\
\text { microrregiões }\end{array}$ & $\begin{array}{c}\text { Grau de desigualdade entre } \\
\text { microrregiões (coeficiente de } \\
\text { Williamson - Vw) }\end{array}$ \\
\hline Rondônia & 08 & 0,12 \\
Acre & 05 & 0,14 \\
Amazonas & 13 & 0,52 \\
Roraima & 04 & 0,18 \\
Amapá & 04 & 0,11 \\
Pará & 22 & 0,96 \\
Tocantins & 08 & 0,26 \\
Média & - & $\mathbf{0 , 3 3}$ \\
Desvio padrão & - & $\mathbf{0 , 3 1}$ \\
Coeficiente de & & $\mathbf{0 , 9 6}$ \\
variação & - & \\
\hline
\end{tabular}

Fonte: IBGE (2013). Resultados da pesquisa.

Revista de Estudos Sociais | Ano 2015, N. 33, V. 17, Pag. 39 
Neste sentido, de forma geral, observa-se que os estados que apresentaram as melhores condições econômicas (Pará e Amazonas), são também os que são caracterizados por maiores índices de desigualdade entre suas microrregiões. Ao contrário disso, nos estados caraterizados por economias pouco dinâmicas, são os que apresentaram os menores graus de desigualdade econômica entre microrregiões. Não se trata, portanto, de riqueza e pobreza, mas de uma maior ou menor participação das microrregiões na economia regional.

De acordo com a Tabela 3, o coeficiente de variação do $V_{w}$ foi de 0,96 , o que indicar alta heterogeneidade no que se refere ao indicador para os estados.

O grau de desigualdade econômica intermunicipal para as microrregiões do Norte do Brasil pode ser verificado no Anexo deste estudo. A média do grau de desigualdade econômica intermunicipal para as microrregiões foi de 0,24, 0 que indica, de forma geral, baixa desigualdade no que tange a participação dos municípios na formação do PIB da microrregião. A microrregião que apresentou o maior grau de desigualdade econômica intermunicipal foi Tucuruí, igual 0,85. Está localizada no estado do Pará, é composta por seis municípios, e representa o 5 maior PIB entre as microrregiões do estado. Entre todas as microrregiões do Norte do país, a microrregião Tucuruí representa o 9ำ maior PIB regional.

A microrregião que apresentou o menor grau de desigualdade econômica foi Boca do Acre, localizada no estado do Amazonas, composta por apenas dois municípios, com grau de desigualdade igual a 0,02, indica pouca disparidade econômica entre seus municípios. Seguindo essas mesmas características, têmse as microrregiões Japurá (AM), Tarauacá (AC) e Sudeste de Roraima (RR), todas com grau de desigualdade econômica intermunicipal de 0,03.

É possível verificar que, a participação dos municípios na composição do PIB das microrregiões não apresenta grandes níveis de disparidades. Isto ocorre pelo fato de que na formação das microrregiões se considerou aspectos de similaridade de ordem econômica, geográfica, social, entre outros, que refletem certa homogeneidade entre os municípios que compõem as respectivas microrregiões. Apesar disso, percebe-se que em algumas microrregiões prevalece um alto grau de desigualdade. Geralmente, são microrregiões localizadas em estados que apresentam maiores níveis de industrialização, como o Pará e Amazonas.

\subsection{Análise Exploratória dos Dados da Desigualdade}

Conforme destacado por Longo (1993), é possível que a desigualdade econômica, em determinada região, possa influenciar o comportamento da mesma variável (ou de outras) e regiões diferentes. Neste sentido, utiliza-se da AEDE para determinar se existe externalidades da desigualdade econômica intermunicipal entre as 64 microrregiões do Norte do Brasil.

Por meio da AEDE, verifica-se a existência de regimes espaciais, identificando padrões de autocorrelação espacial. Além disso, avalia-se a relação do grau de desigualdade econômica entre municípios das microrregiões 
com a variável PIB per capita e o índice de desenvolvimento humano municipal (IDH-M).

A distribuição espacial do grau de desigualdade econômica intermunicipal para as microrregiões pode ser visualizada pela Figura 1. O mapa mostra cinco diferentes intervalos do grau de desigualdade (ver legenda).

É possível observar, que a cor mais escura caracteriza as microrregiões com maiores graus de desigualdade econômica, entre 0,54 e 0,85. Essas microrregiões se encontram localizadas nos estados do Pará e Tocantins. Destaca-se ainda, que nesses dois estados está localizada a maior parte das microrregiões que apresentam grau de desigualdade no intervalo de 0,22 a 0,54.

No estado do Amazonas e Rondônia, apesar de se verificar algumas microrregiões com grau de desigualdade econômica relativamente alta, a maioria das microrregiões apresenta baixa desigualdade econômica entre seus respectivos municípios.

Nos estados do Acre, Roraima e Amapá estão localizadas as microrregiões que apresentam os menores graus de desigualdade econômica intermunicipal, no intervalo de 0,02 a 0,22.

Ainda de acordo com a Figura 1, é possível que existam ocorrências de um padrão espacial da distribuição da desigualdade econômica entre as microrregiões que compõem o território geográfico da região Norte do Brasil.

Figura 1 - Distribuição espacial do grau de desigualdade econômica intermunicipal nas microrregiões do Norte do Brasil, 2010

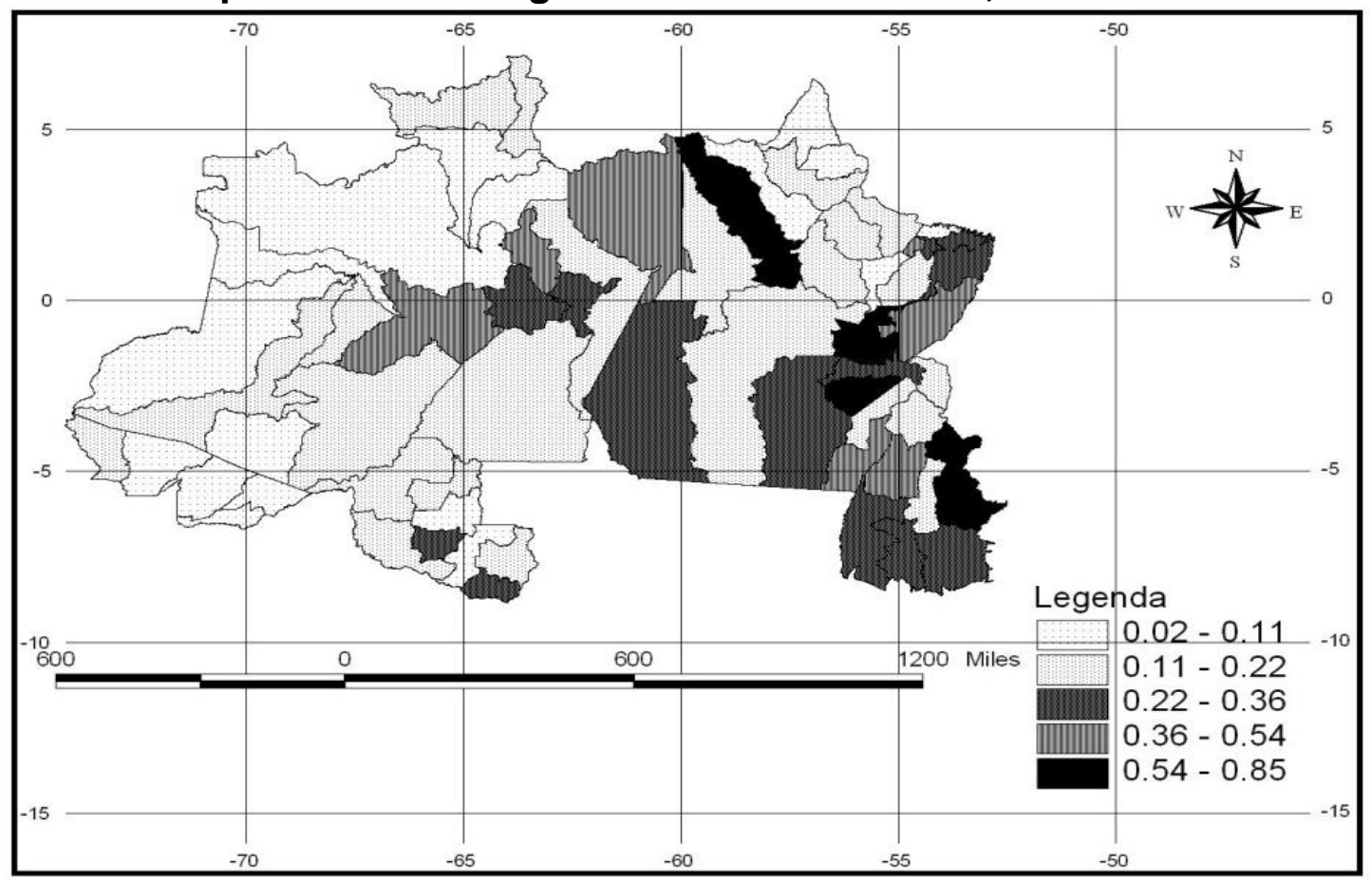

Fonte: Resultados da pesquisa.

Apesar dos indícios da existência de padrões de distribuição, como ressaltado anteriormente, é necessário verificar, por meio das estatísticas, se esses padrões podem ser considerados estatisticamente válidos. Neste sentido,

Revista de Estudos Sociais | Ano 2015, N. 33, V. 17, Pag. 41 
a Tabela 4 apresenta os resultados da estatística I de Moran univariado para o grau de desigualdade econômica intermunicipal nas microrregiões estudadas. Essa estatística pretende confirmar ou não a hipótese de distribuição aleatória dos dados.

Tabela 4 - Indicador de autocorrelação I de Moran univariado global para o grau desigualdade econômica intermunicipal nas microrregiões do Norte do Brasil, em 2010

\begin{tabular}{lcc}
\hline \multirow{2}{*}{ Matriz } & \multicolumn{2}{c}{ Moran } \\
\cline { 2 - 3 } & $\mathbf{I}$ & p-value \\
\hline Rainha & 0,1988 & 0,0093 \\
Torre & 0,2057 & 0,0078 \\
\hline
\end{tabular}

Fonte: Resultados da pesquisa.

Os resultados obtidos referentes ao I de Moran, tanto com utilização da matriz Rainha como no caso da Torre, tiveram o mesmo sentido, ou seja, I > 0, o que indica autocorrelação espacial positiva para o grau de desigualdade econômica nas microrregiões. Isso significa que microrregiões que apresentam um alto (baixo) grau de desigualdade são vizinhas de outras que também ostentam altos (baixo) graus de desigualdade econômica.

A Figura 2 mostra a distribuição espacial do I Moran univariável global para o grau desigualdade econômica intermunicipal nas microrregiões do Norte do Brasil, em 2010.

É possível visualizar, de forma geral, que as microrregiões com altos graus de desigualdade econômica intermunicipal são rodeadas, próximas ou contíguas, de outras que apresentam também altos graus de desigualdade (AltoAlto). Neste caso, microrregiões com altas taxas de desigualdade exercem externalidades negativas sobre suas vizinhas, as quais tendem a apresentar graus de desigualdade elevados.

Da outra forma, microrregiões com baixo grau de desigualdade são rodeadas, próximas ou contíguas de outras que exibem baixos graus de desigualdade (Baixo-Baixo). Neste caso, tem-se externalidade positiva, ou seja, microrregiões com baixo indicador de desigualdade estão rodeadas por outras caracterizadas por baixas taxas de desigualdade.

Existem outros padrões espaciais como no caso em que microrregiões com altos graus de desigualdade econômica intermunicipal estão circunvizinhas de outras que possuem baixos graus de desigualdade intermunicipal (externalidade positiva). E por fim, tem-se o regime espacial em que microrregiões com baixos graus de desigualdade estão localizadas próximas a microrregiões com altos graus de desigualdade econômica intermunicipal (externalidade negativa).

Com o objetivo de identificar agrupamentos de autocorrelação espacial estatisticamente significativos, a Figura 3 mostra o mapa de significância (LISA) do I de Moran global, destacando os clusters espaciais para o grau de desigualdade econômica nas microrregiões consideradas neste estudo.

Ano 2015, N.33, V.17, Pag.42 | Revista de Estudos Sociais 
Figura 2 - Distribuição espacial do I Moran univariável global para o grau desigualdade econômica intermunicipal nas microrregiões do Norte do Brasil, em 2010

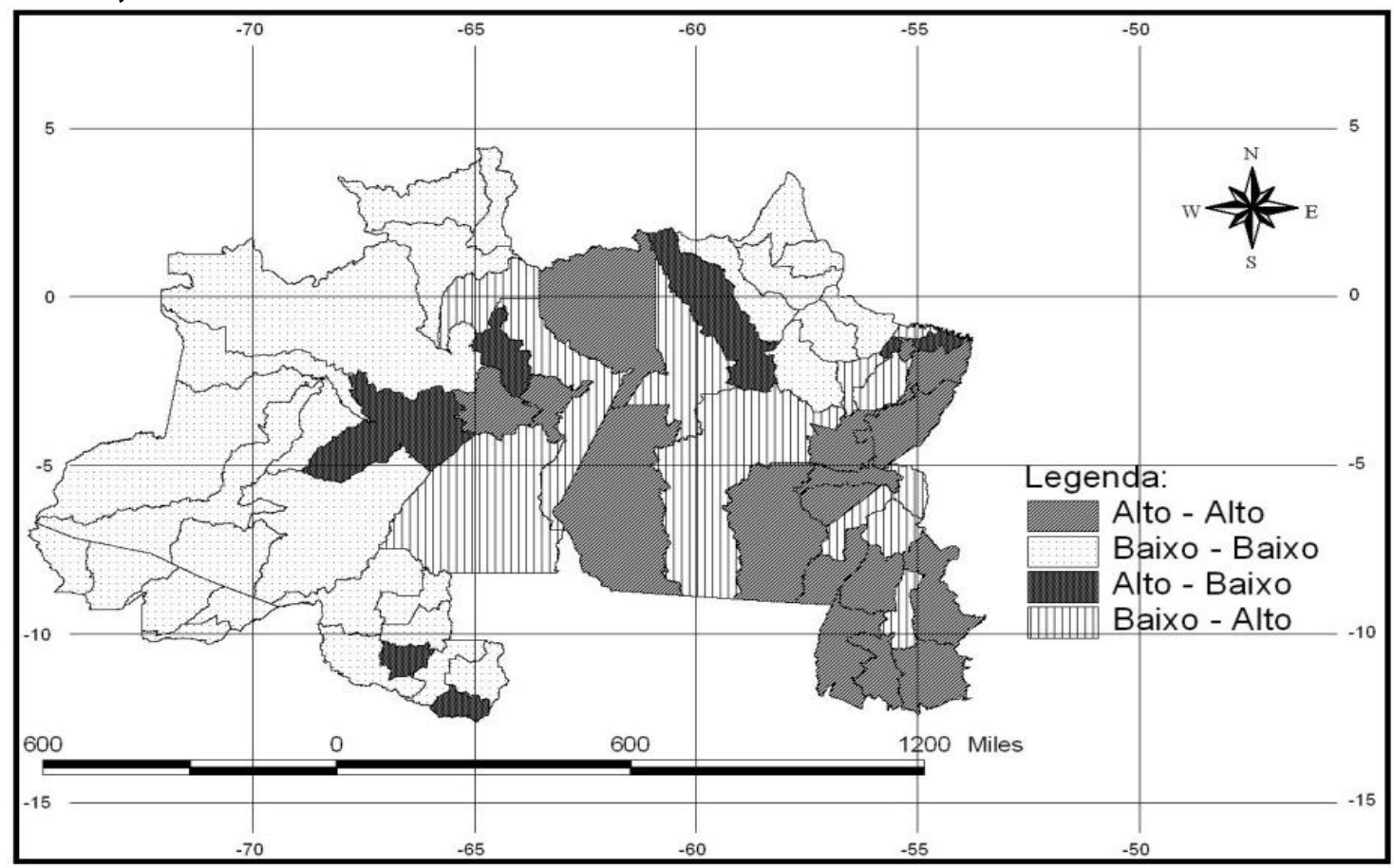

Fonte: Resultados da pesquisa.

Inicialmente, verifica-se que a maior parte das 64 microrregiões analisadas pelo I de Moram não apresenta um regime espacial estatisticamente significante, ou seja, nessas microrregiões, em nível local não pode afirmar e existência de um padrão espacial estatisticamente aceitável.

Porém, observa-se que a estatística local de Moran confirma a tendência de autocorrelação espacial positiva revelada pela estatística global, ou seja, ocorre que algumas microrregiões representam clusters espaciais estatisticamente significantes com características de similaridade do tipo BaixoBaixo, ou seja, microrregiões com baixo grau de desigualdade econômica circunvizinhas de microrregiões com baixo grau de desigualdade econômica.

Nesse grupo se encontram 10 microrregiões, distribuídas da seguinte forma: três no estado do Acre, dois no estado do Amazonas, um no estado de Roraima, dois no estado do Amapá e um no estado do Pará.

Um segundo cluster espacial observado se refere às microrregiões que apresentam características de tipo Baixo-Alto (dissimilaridade). São microrregiões com baixo grau de desigualdade circunvizinhas de microrregiões que apresentam baixo grau de desigualdade. Nesse cluster se encontram um total de quatro microrregiões, duas no estado do Pará e duas no estado de Tocantins.

O terceiro cluster identificado é do tipo Alto-Alto, em que microrregiões com alto grau de desigualdade estão próximas de microrregiões com alto grau 
de desigualdade econômica. Neste grupo estão quatro microrregiões do Pará e uma do Tocantins

Figura 3 - Mapa de significância do I de Moran uni variável global para o grau de desigualdade nas microrregiões da região Norte do Brasil, em 2010

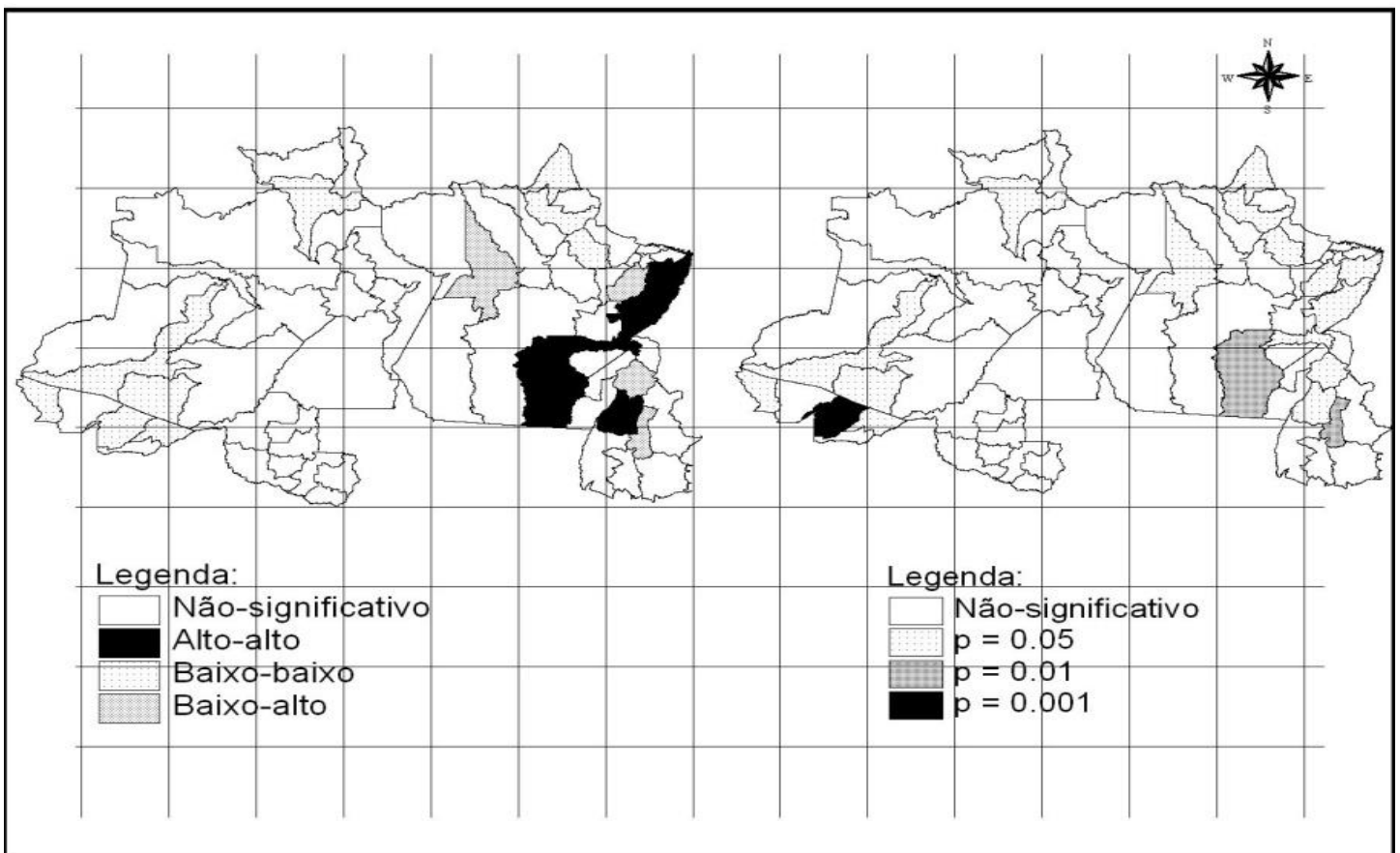

Fonte: Resultados da pesquisa.

.Para verificar a relação, possivelmente existente, entre o grau de desigualdade econômica intermunicipal nas microrregiões com o PIB per capita e o IDH-M, utilização do teste de autocorrelação I de Moran multivariado global. A Tabela 5 mostra os resultados para as variáveis consideradas.

De acordo com a Tabela 5, o I de Moran multivariado global determinado com a utilização da matriz Rainha foi de -0,0101, porém, não significante ( $p$-value $=0,8671$ ). Com a utilização da matriz Torre o I de Moran resultou em -0,0097, também não significante $(\mathrm{p}$-value $=0,9015)$. Em relação ao IDH-M, tanto com a matriz Rainha quanto da Torre o I de Moran foi de 0,090, porém, não significativo $(p$-value $=0,1467)$.

Tabela 5 - I de Moran multivariado global para o grau desigualdade econômica intermunicipal nas microrregiões do Norte do Brasil versus PIB per capita e IDH-M, em 2010

\begin{tabular}{lcccc}
\hline \multirow{2}{*}{ Matriz } & \multicolumn{2}{c}{ PIB per capita } & \multicolumn{2}{c}{ IDH-M } \\
\cline { 2 - 5 } & $\mathbf{I}$ & $\mathbf{p}$-value & I & p-value \\
\hline Rainha & $-0,0101$ & 0,8671 & 0,0901 & 0,1467 \\
Torre & $-0,0097$ & 0,9015 & 0,0901 & 0,1467
\end{tabular}


Neste cenário global das microrregiões analisadas, apesar do resultado do I de Moran multivariado global para o grau de desigualdade versus PIB per capita indicar correlação negativa, e ainda, no caso do grau de desigualdade versus IDH-M indicar correlação positiva, esses resultados não se mostram significativos estatisticamente.

Assim sendo, de acordo com o I de Moram global, não existem clusters espaciais, ou seja, não existe relação espacial entre as variáveis PIB per capita e IDH-M com o indicador de desigualdade utilizado nesse trabalho. Desse modo, não se verifica a existência de externalidade do PIB per capita e IDH-M sobre a desigualdade econômica intermunicipal nas microrregiões.

No entanto, é necessário considerar que as estatísticas globais não são capazes de identificar situações em que ocorram correlações locais estatisticamente significativas. Neste sentido, assim como anteriormente, analisa-se o Indicador Local de Associação Espacial (LISA). As Figuras 4 e 5 mostram o mapa de significância (LISA) em nível local dos resultados da Tabela 5.

Percebe-se na Figura 4, que na relação grau de desigualdade versus PIB per capita, quatro clusters dos tipos: Baixo-Baixo, Alto-Baixo, Baixo-Alto e AltoAlto.

Figura 4 - LISA multivariado para o grau de desigualdade econômica versus PIB per capita nas microrregiões da região Norte do Brasil, em 2010

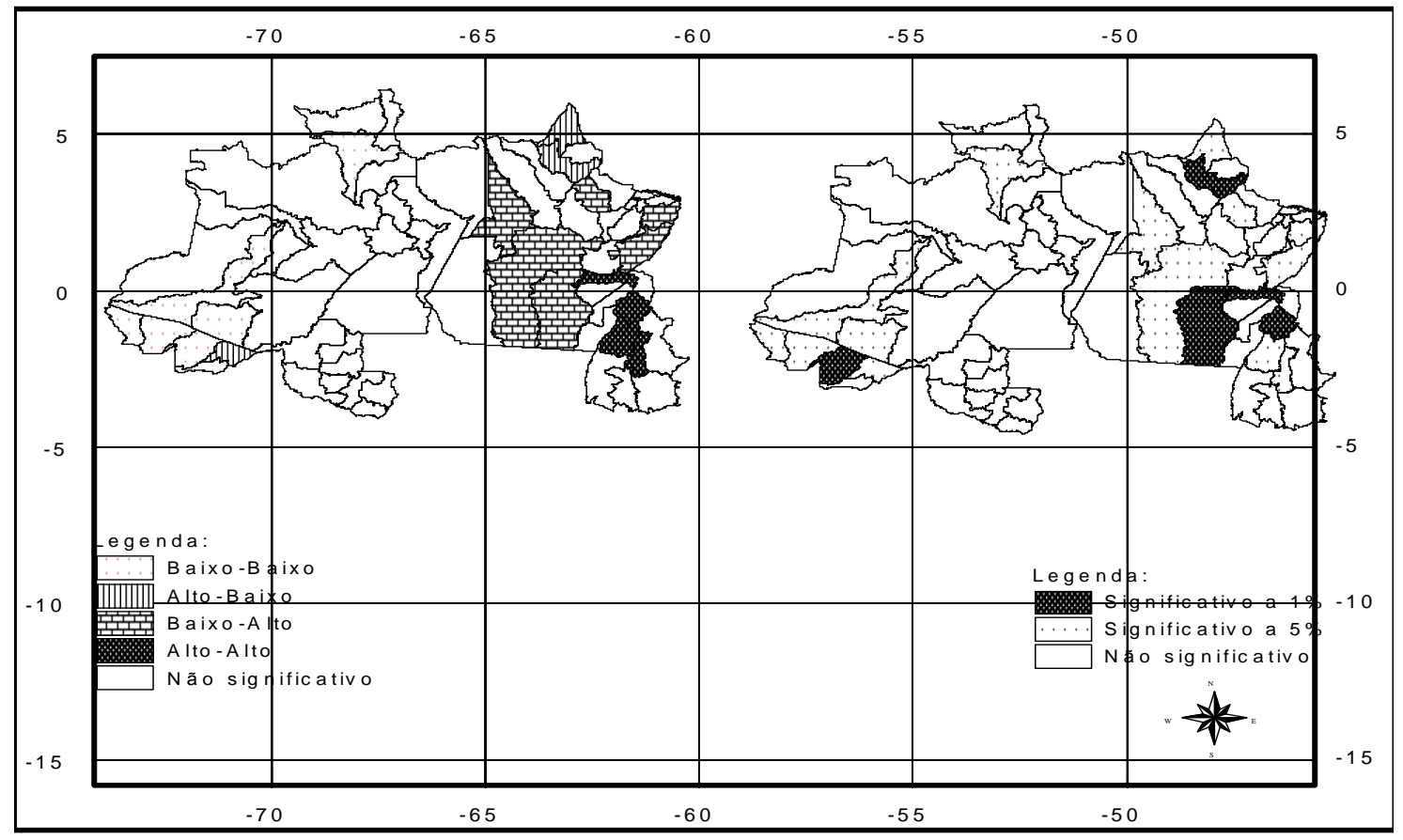

Fonte: Resultados da pesquisa.

É no estado do Pará onde existe o maior número de microrregiões que apresentam relacionamento local entre o grau de desigualdade econômica e 0 PIB per capita. Neste estado, existem sete microrregiões com característica do 
tipo Baixo-Alto. Destaca-se ainda, uma microrregião caracterizada por Alto-Alto, com significância $1 \%$.

A Figura 5 mostra o mapa de significância (LISA) do grau de desigualdade versus o IDH-M. Assim como no caso do PIB per capita têm-se quatro cluster do tipo: Baixo-Baixo, Alto-Baixo, Baixo-Alto e Alto-Alto.

No estado do Amazonas se encontram duas microrregiões com característica Baixo-Baixo. O mesmo ocorre no estado de Roraima. No estado do Amapá verifica-se que apenas uma microrregião tem é caracterizada pelo tipo Alto-Baixo, sendo ainda que, existe uma microrregião neste estado com característica do tipo Alto-Baixo. O estado de Tocantins apresenta duas microrregiões com característica do tipo Alto-Alto.

No estado do Acre observam-se duas microrregiões caracterizadas com Baixo-Baixo. Com essa mesma caracterização verificam-se três microrregiões no Amazonas e uma microrregião no Pará. Isso significa que essas microrregiões por baixo grau de desigualdade econômica são circunvizinhas de outras com que apresentam baixos IDH-M.

Figura 5 - LISA multivariado para o grau de desigualdade econômica versus IDH-M nas microrregiões da região Norte do Brasil, em 2010

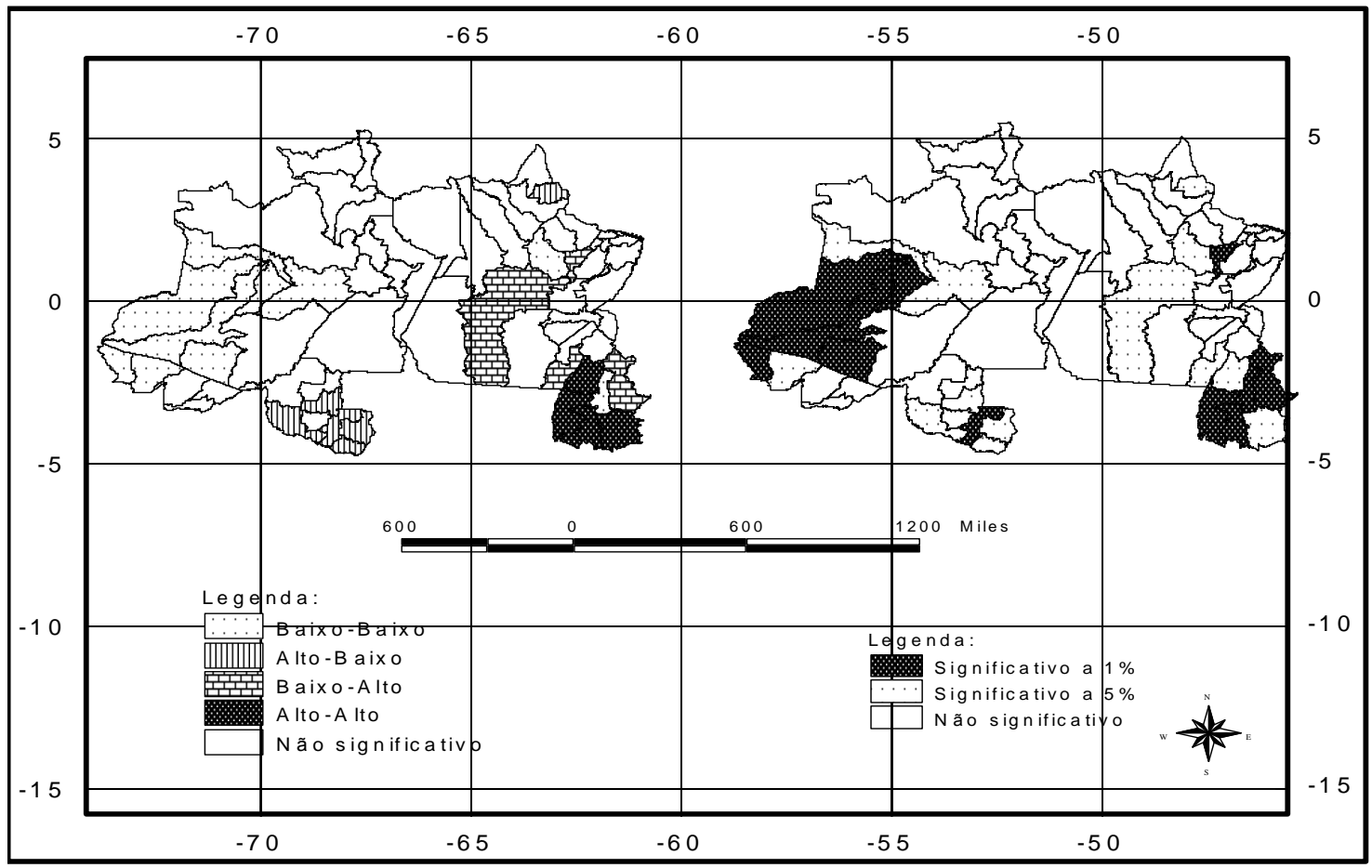

Fonte: Resultados da pesquisa.

De outra forma, no estado de Rondônia observam-se quatro microrregiões com características de Alto-Baixo. Neste mesmo sentido se encontra no estado de Roraima apresenta uma microrregião. Nesses estados, essas microrregiões que apresentam alto grau de desigualdade econômica são circunvizinhas de outras com baixo IDH-M. 
No estado do Pará, três microrregiões apresentam características do tipo Baixo-Alto. Assim como, no Tocantins observa-se uma microrregião caracterizada por Baixo-Alto e outras quatro caracterizadas por Alto-Alto.

De forma geral, as Figuras 4 e 5 mostram que existe correlação entre 0 grau de desigualdade e o PIB per capita, bem como grau de desigualdade e IDHM.

Neste contexto, em algumas microrregiões verifica-se correlação positiva, ou seja, cenários em que microrregiões com alto (baixo) grau de desigualdade são circunvizinhas de outras que apresentam PIB per capita ou IDH-M em valores altos. Porém, é possível que ocorra correlação negativa, em que microrregiões como alto (baixo) grau de desigualdade sejam vizinhas de microrregiões com baixo (alto) valores de PIB per capita ou IDH-M.

\section{CONSIDERAÇÕES FINAIS}

O tema da desigualdade entre as economias regionais tem ocupado cada vez mais espaço no âmbito dos estudos econômicos e sócias. Neste trabalho, investiga-se a respeito da desigualdade econômica intermunicipal na região Norte do Brasil, mais especificamente o estudo se aplica às microrregiões do Norte do país.

Objetiva-se, de forma geral, identificar padrões espaciais de desigualdade econômica intermunicipal entre as microrregiões que compõem a região Norte do Brasil no período de 2010. Para tal, é determinado o grau de desigualdade econômica intermunicipal para as microrregiões com a utilização do Coeficiente de Willianson e, posteriormente, se utiliza a Análise Exploratória de Dados Espaciais para verifica possíveis interações espaciais.

Para contribuir com estudo foi determinado o grau de desigualdade entre as microrregiões para os estados. Os estados que apresentaram as melhores condições econômicas, são também os que são caracterizados por maiores índices de desigualdade entre suas microrregiões. Ao contrário disso, nos estados caracterizados por economias pouco dinâmicas, são os que apresentaram os menores graus de desigualdade econômica entre microrregiões.

O estudo confirma observações empíricas e revela que na Região norte do Brasil ocorre um alto grau de desigualdade entre as microrregiões que a compõem. O grau de desigualdade de Williamson, considerando todas as microrregiões foi de 0,76 .

Esse resultado indica que são grandes as distorções econômicas entre as microrregiões do Norte do Brasil. Esse fenômeno está relacionado diretamente com o fato de que a riqueza produzida na região Norte continua concentrada no espaço geográfico, ou seja, o crescimento econômico da região não se reproduz em melhor distribuição do PIB para microrregiões regiões localizadas na margem do dinamismo econômico.

Revista de Estudos Sociais | Ano 2015, N. 33, V. 17, Pag. 47 
Neste sentido, é conhecido que em regiões de baixa produtividade, no geral, a atividade econômica tende se concentrar no espaço, e é o que se verifica no Norte brasileiro. Grande parte das indústrias, atividades produtivas e mão-deobra qualificada tende a se localizar em poucas microrregiões.

A média do grau de desigualdade econômica intermunicipal para as microrregiões foi de 0,24 . Esse dado indica que a desigualdade econômica é menor quando se aplica a análise as microrregiões. Ou seja, ao se determinar o grau de desigualdade intermunicipal para cada uma das microrregiões observase que existe uma forte homogeneidade na participação de cada município na formação do PIB da microrregião.

De forma geral, percebe-se que, a participação dos municípios na composição do PIB das microrregiões não apresenta grandes níveis de disparidades. Isto ocorre pelo fato de que na formação das microrregiões se considerou aspectos de similaridade de ordem econômica, geográfica, social, e etc..... Que refletem certa homogeneidade econômica entre os municípios que compõem as respectivas microrregiões.

Com a análise espacial é possível verificar a existência de efeitos espaciais de uma microrregião sobre outras e vice-versa. Notadamente, existe dependência espacial no que tange ao grau de desigualdade econômica nas microrregiões.

A estatística do I de Moran univariado global mostra evidencia 04 padrões espaciais. Microrregiões que apresentam altos graus de desigualdade econômica intermunicipal são rodeadas, próximas ou contíguas, com outras que apresentam também altos graus de desigualdade (Alto-Alto). Da mesma forma que microrregiões com baixo grau de desigualdade são rodeadas, próximas ou contíguas de outras que exibem baixos graus de desigualdade (Baixo-Baixo). Observam-se padrões espaciais como no caso em que microrregiões com altos graus de desigualdade econômica intermunicipal que estão circunvizinhas de outras que possuem baixos graus de desigualdade intermunicipal (Alto-Baixo), e por fim, o padrão Baixo-Alto, em que microrregiões com baixos graus de desigualdade estão localizadas próximas a microrregiões com altos graus de desigualdade econômica intermunicipal.

Neste aspecto, é possível perceber efeitos de externalidades, positivas e negativas, de regiões que apresentam alto grau de desigualdade em microrregiões que exibem baixo grau de desigualdade e vice-versa. Desta forma, fica claro que a distribuição do grau de desigualdade econômica intermunicipal para as microrregiões do Norte, não se dá de forma aleatória.

O LISA revela a predominância das microrregiões que se agrupam em um cluster com características de similaridade do tipo Baixo-Baixo, ou seja, microrregiões com baixo grau de desigualdade econômica circunvizinhas de microrregiões com baixo grau de desigualdade econômica. Um segundo cluster observado se refere às microrregiões que apresentam características de tipo Baixo-Alto (dissimilaridade), ou seja, microrregiões com baixo grau de desigualdade econômica circunvizinhas de microrregiões que apresentam baixo grau de desigualdade econômica.

A análise espacial, por meio da estatística global, revela ainda, que a relação entre o grau de desigualdade nas microrregiões versus o PIB per capita 
não é significativo estatisticamente, ou seja, não existe nenhum padrão espacial de correlação entre essas variáveis. Este mesmo fenômeno pode ser verificado para a relação entre o grau de desigualdade econômica nas microrregiões e o IDH-M.

A estatística local indica que em algumas microrregiões ocorre correlação positiva, ou seja, cenários em que microrregiões com alto (baixo) grau de desigualdade são circunvizinhas de outras que apresentam PIB per capita ou IDH-M em valores altos. Porém, é possível que ocorra correlação negativa, em que microrregiões como alto (baixo) grau de desigualdade sejam vizinhas de microrregiões com baixo (alto) valores de PIB per capita ou IDH-M.

De modo geral, com algumas exceções já destacadas, a desigualdade econômica intermunicipal nas microrregiões é considerada, em certa medida, pequena, haja vista que os municípios que compõem as microrregiões foram agrupados considerando aspectos comuns. Porém, não se pode desprezar o fato de qualquer nível de desigualdade econômica constitui um entrave para o desenvolvimento regional.

A análise espacial ajuda a compreender de que forma a desigualdade de distribui no espaço. Neste sentido, ficou claro que existe um padrão de distribuição do grau de desigualdade entre as microrregiões do Norte do país, ou seja, em certa medida, pode-se afirmar que em alguns casos ocorre dependência pendencia espacial.

Este estudo está limitado à análise da região Norte, considerando especificamente as microrregiões. Assim sendo, não se esgota a pesquisa a respeito do tema principal. Portanto, recomenda-se a análise mais ampla, considerando as demais grandes regiões do Brasil e suas respectivas microrregiões, a fim de obter dados comparativos com os obtidos para a região aqui estudada.

\section{RFERÊNCIAS}

ALMEIDA, E.S. Curso de econometria espacial aplicada. Piracicaba, 2004.

ALMEIDA, E.S.; PACHECO, G.O.; PATROCÍNIO, A.P.B.; DIAS, S.M. Produtividade do café em Minas Gerais: uma análise espacial. In: CONGRESSO BRASILEIRO DE ECONOMIA E SOCIOLOGIA RURAL, Fortaleza, 2006. Anais... Brasília: SOBER, 2006.

ALMEIDA, E. Econometria espacial aplicada. Campinas: Alínea, 2012.

ANDRADE, M.C.; ANDRADE, S.M.C.A federação brasileira: uma análise geopolítica e geo-social.2.ed. São Paulo: Contexto, 2003.

ANSELIN, L. Spacestat tutorial: a workbook for using Spacestat in the analysis of spatial data. Urbana Champaign, Illinois, 1992.

ANSELIN, L. Local indicators of spatial association-LISA. Geographical Analysis,v. 27, n. 2, 1995.

Revista de Estudos Sociais | Ano 2015, N. 33, V. 17, Pag. 49 
BALDWIN, R.; MARTIN, P. Aglomeração e crescimento regional. In: CARVALHO, A.X. et al. Ensaios de economia regional e urbana.Brasília: IPEA, 2007.

CARVALHO, Terciane Sabadini. A hipótese da curva de Kuznets ambiental e - Protocolo de Quioto. 2009. Dissertação (Mestrado em Economia) Universidade Federal de Juiz de Fora, Juiz de Fora, MG.

EATON, B.C.; EATON, F.D. Microeconomia. 3.ed. São Paulo: Saraiva, 1999.

FUJITA, M.; THISSE, J. Economics of agglomeration: cities, industrial location and regional growth. Cambridge: Cambridge University Press, 2002.

HIRSCHMAN, A. The strategy of economic development. New Haven: Yale University, 1961.

KRUGMAN, P. Increasing returns and economic geography. Journal of Political Economy, v. 99, p. 483-499, 1991.

LONGO, Carlos Alberto. Economia do setor público. São Paulo: Atlas, 1993.

LORENA, Rodrigo Borrego; BERGAMASCHI, Rodrigo Bettin; Gilmar dos Reis Leite. Análise exploratória espacial do Índice de Desenvolvimento Humano Municipal do Estado do Espírito Santo. In: SIMPÓSIO BRASILEIRO DE SENSORIAMENTO REMOTO, 15, 2011, Curitiba. Anais... São Paulo: INPE, 2011.

MAGALHÃES, A.; HEWINGS, G.; AZZONI, C. Spatial dependence and regional convergence in Brazil. Chicago: University of Illinois, 2000. 20 p. (Working Papers, 11).

MARTINHO, Vitor João Pereira. Análise dos efeitos espaciais na produtividade setorial entre as regiões portuguesas. 2005. (Discussion Paper, 30).

MIRDAL, G. Economic theory and under-developed regions. London: Duckworth, 1960.

PINDYCK, Robert S.; RUBINFELD, D.L. Microeconomia. 2.ed. São Paulo: Makron Books, 1994.

RAMOS, F.R. Análise espacial de estruturas intra-urbanas: o caso de São Paulo. 2002. 139 f. Dissertação (Mestrado em Sensoriamento Remoto) Instituto Nacional de Pesquisas Espaciais, São José dos Campos, SP.

SILVA, R.G.; LÍRIO, V.S.; LIMA, J.E.Externalidade. In: SANTOS, M.L.; VIEIRA, W.C.; LíRIO, V.S.Microeconomia aplicada. Visconde do Rio Branco, MG: Suprema, 2009. 